\title{
A Biometric Identification System Based on Eigenpalm and Eigenfinger Features
}

\author{
Slobodan Ribaric, Member, IEEE, and Ivan Fratric
}

\begin{abstract}
This paper presents a multimodal biometric identification system based on the features of the human hand. We describe a new biometric approach to personal identification using eigenfinger and eigenpalm features, with fusion applied at the matching-score level. The identification process can be divided into the following phases: capturing the image; preprocessing; extracting and normalizing the palm and strip-like finger subimages; extracting the eigenpalm and eigenfinger features based on the K-L transform; matching and fusion; and, finally, a decision based on the ( $\mathrm{k}, \mathrm{l})$-NN classifier and thresholding. The system was tested on a database of 237 people ( 1,820 hand images). The experimental results showed the effectiveness of the system in terms of the recognition rate (100 percent), the equal error rate (EER $=0.58$ percent), and the total error rate $(T E R=0.72$ percent).
\end{abstract}

Index Terms-Biometrics, multimodal systems, hand-based identification, K-L transform, eigenpalms, eigenfingers.

\section{INTRODUCTION}

B IOMETRICS is an emerging technology [1], [2] that is used to identify people by their physical and/or behavioral characteristics and, so, inherently requires that the person to be identified is physically present at the point of identification. The physical characteristics of an individual that can be used in biometric identification/verification systems are fingerprint [3], [4], hand geometry [5], [6], palm print [7], [8], [9], face [4], [10], iris [11], [12], retina [13], and ear [14]; the behavioral characteristics are signature [2], lip movement [15], speech [16], keystroke dynamics, gesture, and gait [1], [2]. Biometric systems based on a single biometric characteristic are referred to as unimodal systems. They are usually more cost-efficient than multimodal biometric systems. However, a single physical or behavioral characteristic of an individual can sometimes fail to be sufficient for identification. For this reason, multimodal biometric systems -i.e., systems that integrate two or more different biometric characteristics-are being developed to provide an acceptable performance, to increase the reliability of decisions, and to increase robustness to fraudulent technologies [17]. The biometric community puts a lot of effort into working on technical standards in the field of biometric fusion [18].

Although systems based on fingerprints and eye features have, so far at least, achieved the best matching performance, the human hand also contains a wide variety of features -e.g., shape, texture, and principal palm lines-that can be used by biometric systems. These features of the human hand are relatively stable and the hand image from which they are extracted can be acquired relatively easily. Furthermore, it has been reported [1], [19] that identification systems based on hand features are the most acceptable to users.

- The authors are with the Department of Electronics, Microelectronics, Computer, and Intelligent Systems, Faculty of Electrical Engineering and Computing, University of Zagreb, Unska 3, 10000 Zagreb, Croatia.

E-mail: \{slobodan.ribaric, ivan.fratric\}@fer.hr.

Manuscript received 4 Feb. 2004; revised 22 Nov. 2004; accepted 14 Feb. 2005; published online 14 Sept. 2005.

Recommended for acceptance by $R$. Chellappa.

For information on obtaining reprints of this article, please send e-mail to: tpami@computer.org, and reference IEEECS Log Number TPAMI-0067-0204.
Our multimodal biometric identification system is based on features extracted from hand images by means of the Karhunen-Loeve (K-L) transform. Features extracted by projecting palm images into the subspace obtained by the K-L transform are called eigenpalm features, whereas those extracted by projecting strip-like images of fingers are called eigenfinger features. Fusion at the matching-score level is used. The final decision (the user is identified or rejected) is based on the modified k-NN rule and thresholding.

The rest of the paper is organized as follows: Section 2 presents related work in the field of palm print, finger, and hand-geometry-based biometric systems, biometric systems using the K-L transform, and information fusion in multimodal biometric systems. An overview of our system is given in Section 3. Section 4 deals with the problem of template generation. In Section 5, matching and fusion at the matchingscore level are described. The experimental results are reported in Section 6. The conclusions and future work are presented in Section 7.

\section{Related Work}

Most biometric systems that use the human hand are based on either palm or hand-geometry characteristics. Palm printbased biometric systems normally use the following characteristics: prominent palm-line features, the end points of these lines, texture, global texture energy, or some combination of these characteristics [7], [8], [9], [20], [21], [22].

Hand-geometry-based systems usually involve determining the lengths and widths of the hands and fingers at different points [5], [6], [23]. Most of these systems use handposition constraints, such as pegs. In [24], a system based on deformable shape matching is proposed. A number of commercial systems based on hand geometry [25] and palm prints [26], [27] are available.

K-L-transform-based techniques have been widely used in the field of biometrics, particularly in face-recognition techniques (eigenfaces) [28], but they have also been used for lip tracking (eigenlips) [15] and hand-gesture recognition through hand contours (eigencontours) [29]. In a recent paper [30], Lu et al. also use eigenpalms for palm print recognition. The images of the palm prints are captured at a resolution of 
$484 \times 384$ pixels. They are then aligned and their size is normalized. From these images, the palm subimages with a fixed size $(128 \times 128$ pixels) are extracted and transformed using the K-L transform. The extraction of the features involves the proposed eigenspace method with featurevector lengths of 50,100,150, and 200. These features can then be clustered by using the weighted Euclidean distance. The test database consists of 191 people, each of whom provided eight images of their left palm and eight images of their right palm. Experiments using different numbers of training samples and different lengths of the feature vector are described. The best recognition rate of 99.149 percent is achieved for four training samples and 100 features. Error rates of FRR $=1$ percent and FAR $=0.03$ percent were also reported.

In general, multimodal biometric systems require integration schemes to fuse the information obtained from the individual modalities. Various levels of fusion are possible [31], [32]:

1. Fusion at the feature-extraction level, where the features extracted using two or more sensors are concatenated.

2. Fusion at the matching-score level, where the matching scores obtained from multiple matchers are combined.

3. Fusion at the decision level, where the accept/reject decisions of multiple systems are consolidated.

In papers [33], [34], the fusion of hand-geometry and palm print features at the matching-score and decision levels are described. Jain and Ross [35] describe an interesting approach to the realization of a multimodal biometric verification system based on face, fingerprint, and hand-geometry features that uses fusion at the matchingscore level based on learning user-specific matching thresholds as well as the weights of an individual biometric trait. Some other references related to fusion at the matchingscore and decision levels are [36], [37], [38], [39].

\section{System Description}

Fig. 1 shows the block-diagram of the proposed multimodal biometric identification system based on the fusion of eigenpalm and eigenfinger features at the matching-score level. In the image-acquisition phase, a hand image is taken using a low-cost scanner. The spatial resolution of the images is 180 dots per inch (dpi)/ 256 gray levels. In the preprocessing module, some standard image-enhancement procedures are applied. Based on the hand contour and reference (stabile) points, the six regions of interest are localized: a square palm region and five strip-like regions of the fingers. Geometry normalization is applied to the original gray-scale hand image for each region of interest to obtain the corresponding subimages. After that, the lighting normalization is performed using histogram fitting. The normalized palm print subimage and five normalized strip-like finger subimages are transformed by the Karhunen-Loeve (K-L) transform into six feature spaces called "eigenpalms space" and "eigenfingers space," respectively. The feature spaces are spanned by a certain number of the largest eigenvectors of the corresponding covariance matrices. The dimensionality of the feature spaces is determined during the training phase of the system. The outputs of the feature-extraction modules, for the sample $\mathbf{x}$, are six feature vectors: the palm-feature vector $\mathbf{P}_{\mathbf{x}}$ and five finger-feature vectors $\mathbf{F}_{\mathbf{x}}^{\mathbf{i}} ; i=1,2, \ldots 5$. The matching between the corresponding vectors and the templates from a database is performed in the subsequent six matching modules. After the normalization of the matcher's outputs, fusion at the matching-score level is obtained by means of the total similarity measure. In the decision module, the $(k, 1)-\mathrm{NN}$ rule, with $\mathrm{k}=1=3$, is used to establish identity, and after thresholding based on the total similarity measure, a final decision is made (the user is identified or rejected).

\section{Template Generation}

The template, as a mathematical representation of the biometric data in our system, consists of six feature vectors: the palm-feature vector $\mathbf{P}_{\mathbf{x}}$ and five finger-feature vectors $\mathbf{F}_{\mathbf{x}}^{\mathbf{i}} ; \mathrm{i}=1,2, \ldots 5$, where $\mathbf{F}_{\mathbf{x}}^{\mathbf{1}}$ represents the little finger, $\mathbf{F}_{\mathbf{x}}^{\mathbf{2}}$ represents the ring finger, $\mathbf{F}_{\mathrm{x}}^{3}$ represents the middle finger, $\mathbf{F}_{\mathrm{x}}^{4}$ represents the index finger, and $\mathbf{F}_{\mathrm{x}}^{5}$ represents the thumb. In this section, the template-generation process is described.

\subsection{Image Capturing, Preprocessing, and Subimage Extraction}

Images of the right hand are scanned at $180 \mathrm{dpi} / 256$ gray levels using a low-cost scanner. The user puts his/her hand on the scanner with the fingers spread naturally; there are no pegs, or any other hand-position constrainers. An example of an image (biometric sample) acquired with this method is shown in Fig. 2a.

The acquired image is binarized using thresholding. Due to the high contrast between the background and the hand on the images, global thresholding provides satisfactory results. Fig. $2 \mathrm{~b}$ shows a binarized image from Fig. 2a. A contour-following algorithm is applied to a binarized image to extract the hand contour. The hand contour is then processed in order that the relevant points for finding regions of interest on the original gray-scale image (the palm region and the finger-strip regions) are determined. An example of a processed hand contour with the relevant points marked is shown in Fig. 3a.

Points T1, T2, T3, T4, T5, B2, B3, B4, and B6 (the fingertips and the valleys between the fingers) are located using the local minima and maxima on the contour. Points B1, B5, and B7 are set by making sure that the distance between each of these points and its corresponding fingertip is the same as the distance between the fingertip and the base-point on the other side of the finger. Points P1 and P2, which are relevant for determining the palm region, are located as shown in Fig. 3a.

The palm is the inner surface of the hand between the wrist and the fingers. In our system, the palm region (the region of interest) is defined as a square region with two of its corners placed on the middle-points of the line segments P1-B2 and B4-P2 (see Fig. 3a).

In order to find the finger-strip region (the region of interest) of each finger, the following steps are performed: Four additional points are determined on the finger contour. Two of them (points F1 and F2; Fig. 3b) are at one-third of the distance between the fingertip and the base of the finger (defined as the middle-point of the line segment B1-B2; Fig. 3b); the other two (points F3 and F4; Fig. 3b) are at twothirds of the same distance. The line connecting the middlepoints of the line segments F1-F2 and F3-F4 defines the line of symmetry for the finger-strip region. The length of the strip is chosen to be five-sixths of the length of the finger (the distance between the base of the finger and the fingertip). Strip widths 


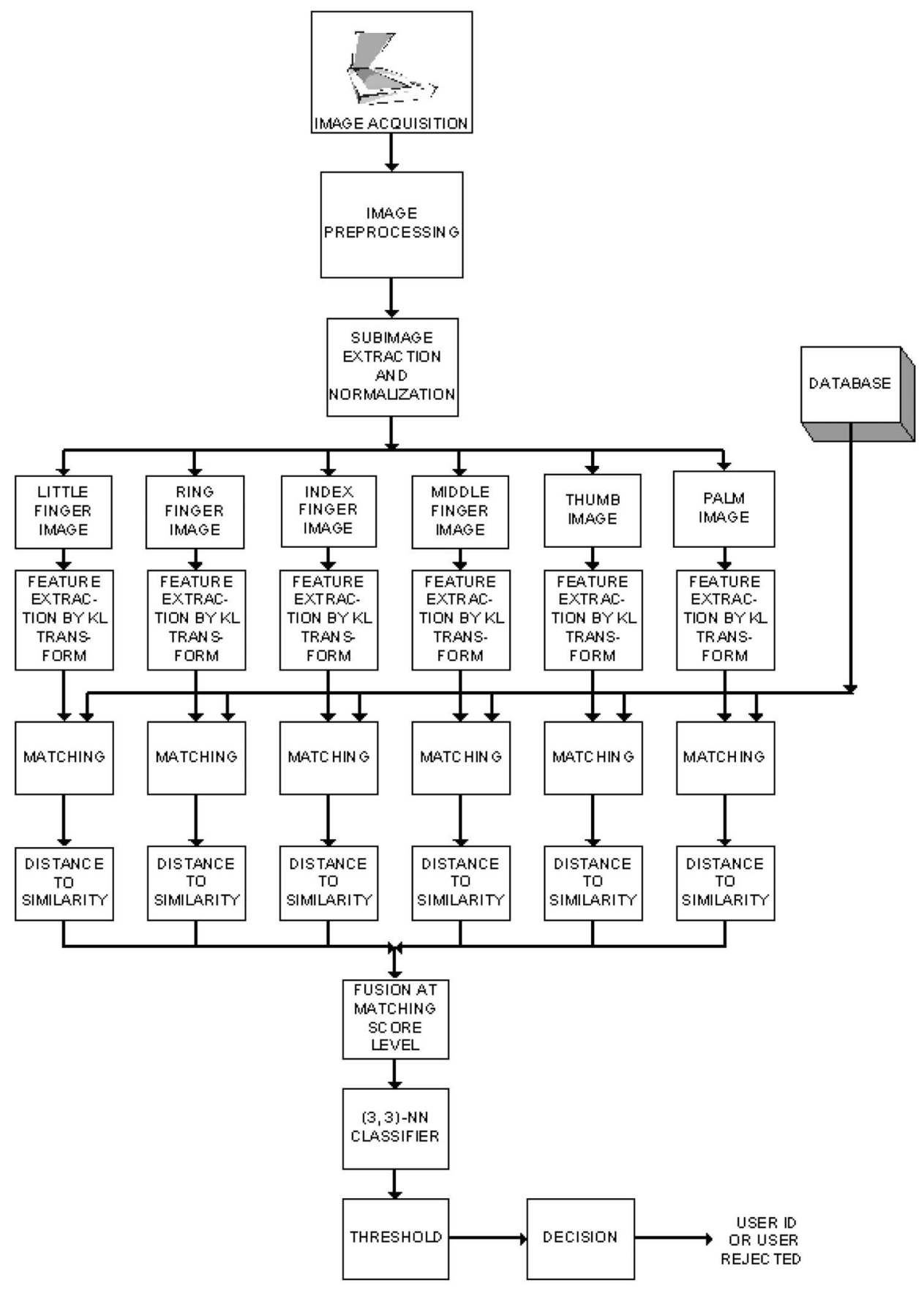

Fig. 1. Block-diagram of the implemented system.

are determined for each finger experimentally, in such a way that the strip's length-width ratio is constant for each finger.

The selected finger-strip region contains the folds of the skin corresponding to the places between the phalanxes of the finger. These folds of the skin and their positions contribute to the discriminatory characteristics of the region.

\subsection{Geometry and Lighting Normalization}

The regions of interest in the original gray-scale images vary in size and orientation from image to image. However, in order to apply the K-L transform, they all need to be normalized to exactly the same size and orientation. Geometry normalization is applied to the gray-scale image to obtain the corresponding hand-part subimages: The palm subimage is normalized to $64 \times 64$ pixels, the little-finger and thumb subimages to $16 \times 64$ pixels, and the ring, middle, and indexfinger subimages to $14 \times 64$ pixels. Fig. 4 a shows the original hand image with the regions of interest marked on it. Figs. $4 b$, $4 \mathrm{c}, 4 \mathrm{~d}, 4 \mathrm{e}, 4 \mathrm{f}$, and $4 \mathrm{~g}$ show the corresponding subimages obtained using the geometry normalization procedure.

After the subimages have been extracted, a lighting normalization using histogram fitting is applied. In this process, a target histogram $G(l)$ is selected for each of the six subimage classes as the histogram of the corresponding subimage obtained from the first hand image in the database. The subimages are transformed so that their original histograms (described with a histogram function $H(l), l=0,1,2, \ldots, 255$; where $l$ is the discrete gray-scale intensity level) become the same as the target histograms (described with a histogram function $G(l)$ ). 


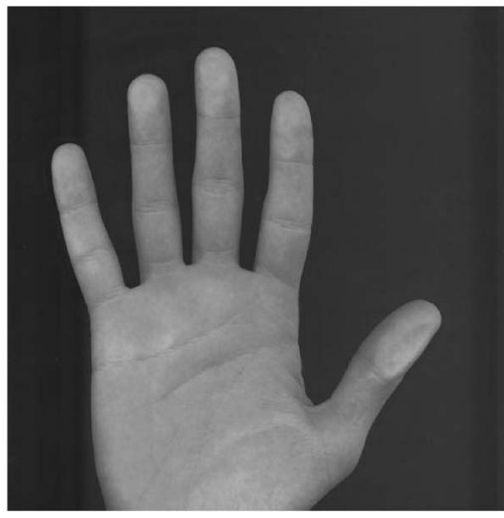

(a)

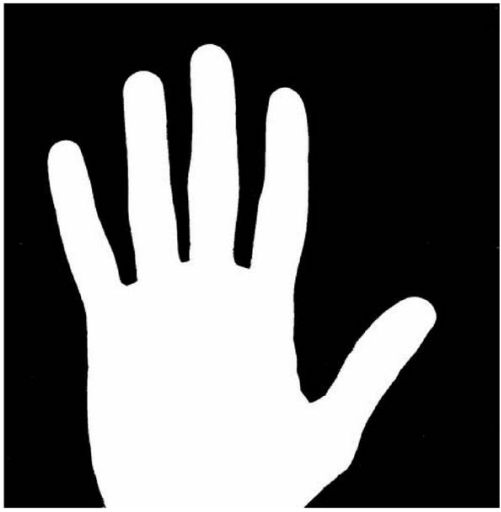

(b)

Fig. 2. (a) Example of a scanned hand image. (b) Binarized hand image.

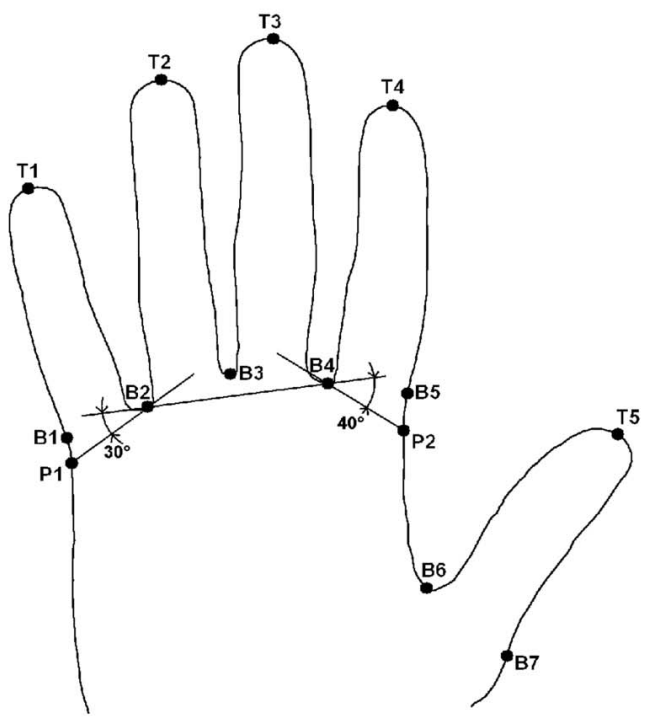

(a)

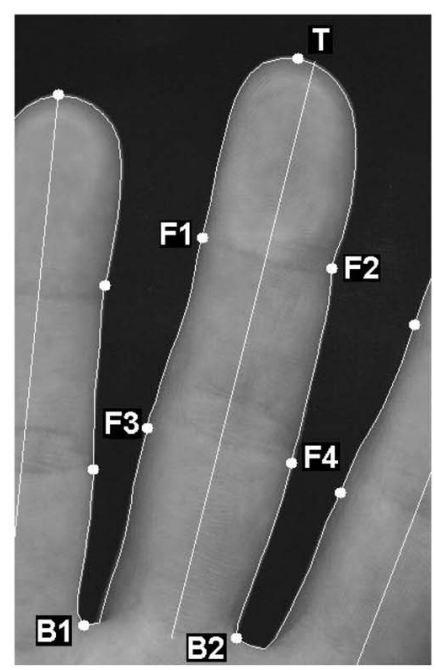

(b)

Fig. 3. (a) The hand contour and the relevant points for finding the regions of interest. (b) Processed finger on the hand contour.
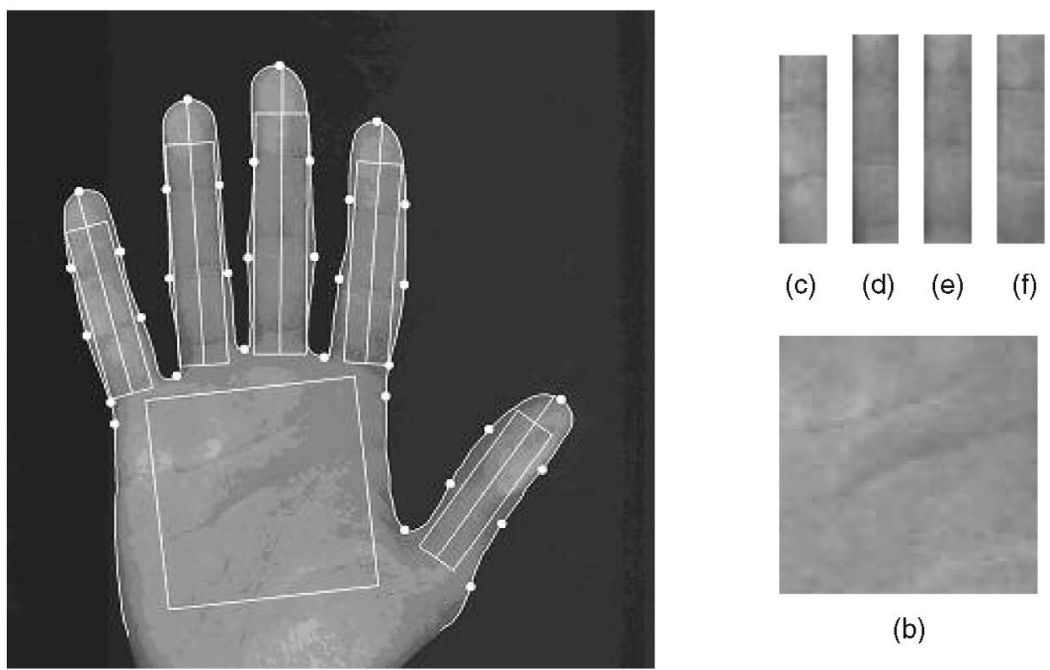

(c) $\quad$ (d) $\quad(e) \quad$ (f)

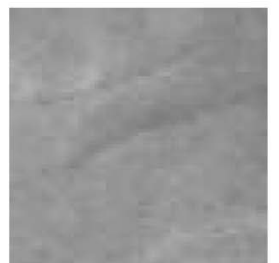

(b)

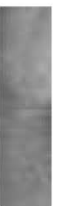

(g)

(a)

Fig. 4. (a) Original hand image with the regions of interest marked on it. (b) Palm subimage. (c) Little-finger subimage. (d) Ring-finger subimage. (e) Middle-finger subimage. (f) Index-finger subimage. (g) Thumb subimage. 

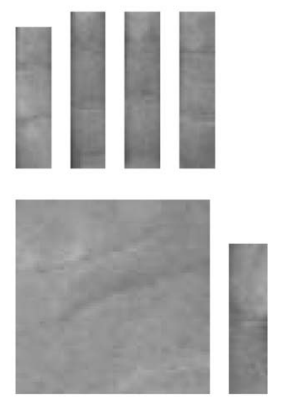

(a)

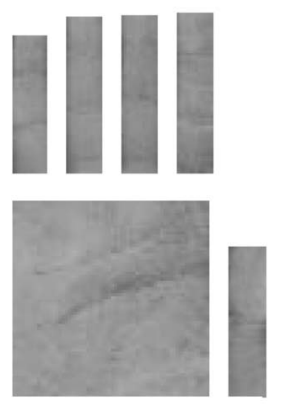

(b)
Fig. 5. Subimages (a) before and (b) after the histogram fitting.

The procedure of histogram fitting [40] can be described as follows: First, we need to find the functions $f_{H \rightarrow U}(l)$ and $f_{G \rightarrow U}(l)$ that map the histograms $H(l)$ and $G(l)$ onto a uniform distribution histogram $U(l)$ :

$$
\begin{array}{r}
f_{H \rightarrow U}(l)=\frac{\sum_{j=0}^{l} H(j)}{\sum_{j=0}^{L-1} H(j)} \\
f_{G \rightarrow U}(l)=\frac{\sum_{j=0}^{l} G(j)}{\sum_{j=0}^{L-1} G(j)},
\end{array}
$$

where $L$ represents the number of discrete intensity levels in the images (256 in our case).

To find the desired mapping function $f_{H \rightarrow G}(l)$ that maps the image histogram onto the target histogram, we need to find the inverse function of $f_{G \rightarrow U}(l)$, which we will mark as $f_{U \rightarrow G}(l)$. The function $f_{U \rightarrow G}(l)$ is simply implemented by searching through all the values of the function $f_{G \rightarrow U}(l)$. After we have this function, we can express the desired mapping $f_{H \rightarrow G}(l)$ as

$$
f_{H \rightarrow G}(l)=f_{U \rightarrow G}\left(f_{H \rightarrow U}(l)\right), l=0,1, \ldots, L-1 .
$$

The experimental results showed that histogram fitting improves the performance of our system in such a way that the recognition error is reduced. We also tested a lighting normalization procedure that involves prespecified mean and variance, as used in [34] for palm print images, but we found that this procedure does not improve the performance of the system as much as histogram fitting.

Fig. 5 presents subimages from Fig. 4, before and after the histogram fitting. Fig. 6 shows some palm subimages with the corresponding histograms from before and after the histogram fitting.

Image preprocessing, subimage extraction, and normalization are based on simple and well-known algorithms. The processing time for all of the above procedures is 0.536 seconds (on a computer with 1177 SPECint_base2000) for a $1,530 \times 1,650$ pixels image.

\subsection{Extraction of the Eigenpalm and Eigenfinger Features}

The eigenspace technique has been widely used for pattern recognition, as well as in the field of biometrics. It is most popular as a face-recognition technique [28], but, in a recent paper [30], it is also used for recognizing palm print images. The K-L transform, also known as PCA (Principal Component Analysis), applied to a set of images, can be used to find the subspace that is occupied by all of the images from the analyzed set. When the images are encoded into this subspace and then returned to the original space, the error between the reconstructed and the original images is minimized (i.e., no other transform exists that can represent these images with the same number of samples and has a smaller reconstruction error).

Eigenpalm and eigenfinger feature extraction is based on the K-L transform and is used to obtain the most important features from the palm and finger subimages in our system. These features are obtained by projecting the original subimages into corresponding subspaces. We create six image subspaces: one for the palm subimages and one for the subimages of each finger. The process of obtaining these subspaces and projecting the subimages into them is identical for all subspaces.

To begin with, we have a training set of $N$ subimages, and each subimage consists of $n$ elements. For example, in the database used to compute eigenspaces we have $N=550$ images. The palm subimages have $n=64 \times 64=$ 4,096 elements, the little-finger and thumb subimages $n=$ $16 \times 64=1,024$ elements, and the ring, middle, and indexfinger subimages have $n=14 \times 64=896$ elements.

The process of obtaining a single subspace [28], [41] consists of finding the covariance matrix $C$ of the training set of subimages and computing its eigenvectors $v_{k} ; k=1,2, \ldots, N$.

The eigenvectors $v_{k}$ corresponding to the largest eigenvalues $\lambda_{k}$ span the base of the sought subspace. Each original subimage can be projected into this subspace as

$$
\eta_{k}=v_{k}^{T} \cdot \Phi_{S} \quad k=1,2, \ldots, m,
$$

where $m$ is the chosen dimensionality of the subimage subspace and $\Phi_{\mathrm{S}}=\Gamma_{\mathrm{S}}-\Psi$, where $\Gamma_{\mathrm{S}}$ is an original subimage from the set of subimages that have to be projected and $\Psi$ is the average subimage of the training set. In Fig. 7, the average palm subimage and the average finger subimages obtained from our training set are presented. The coordinates of the projected images in that subspace, $\eta_{k} ; k=1, \ldots, m$, can be used as a feature vector for the matching procedure.

The eigenvectors spanning the palm-space can be represented as images with the same dimensionality as the palm subimages used to obtain these eigenvectors. These subimages are called eigenpalms. The same procedure for each finger gives us the eigenfingers. Fig. 8 shows the eigenpalms and eigenfingers obtained from the training database. They are presented in decreasing order of the appropriate eigenvalue values. The ordinal numbers of the eigenpalms or eigenfingers are indicated below each image. From Fig. 8, it is obvious that the images with the smaller ordinal numbers (i.e., the larger corresponding eigenvalues) look similar to the $\mathrm{palm} /$ finger images and, so, represent useful information for the image encoding. On the other hand, images with larger ordinal numbers look like noise and do not represent useful information. This is the reason why only eigenvectors corresponding to the large eigenvalues are used for the feature extraction.

The same observations presented in [28] for eigenfaces can also be applied to eigenpalms and eigenfingers: Each 

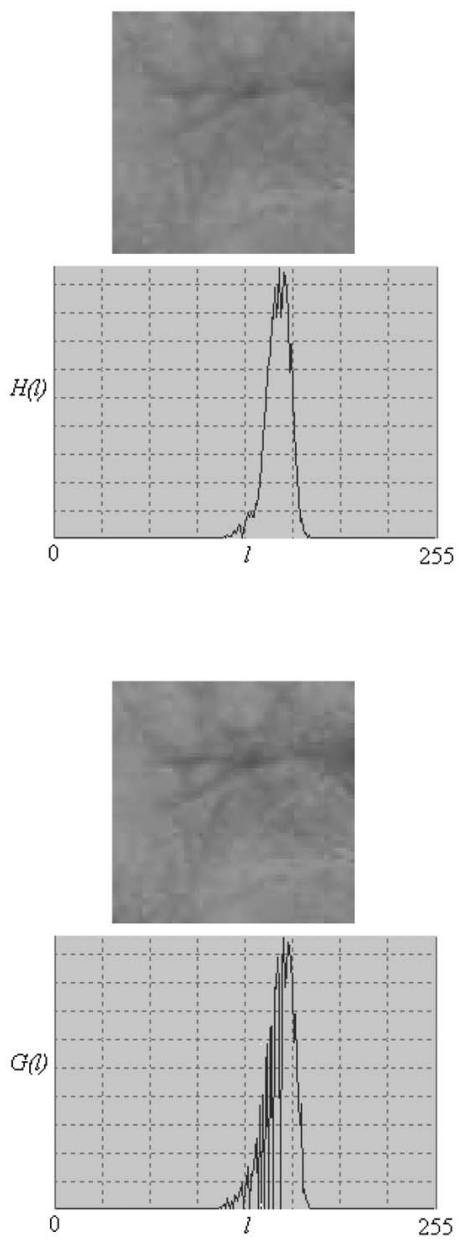

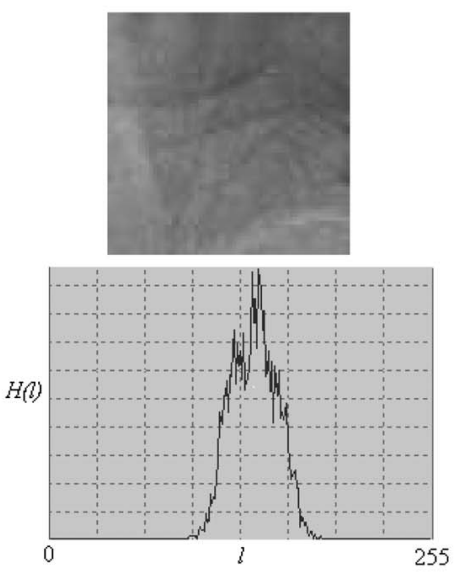

(a)
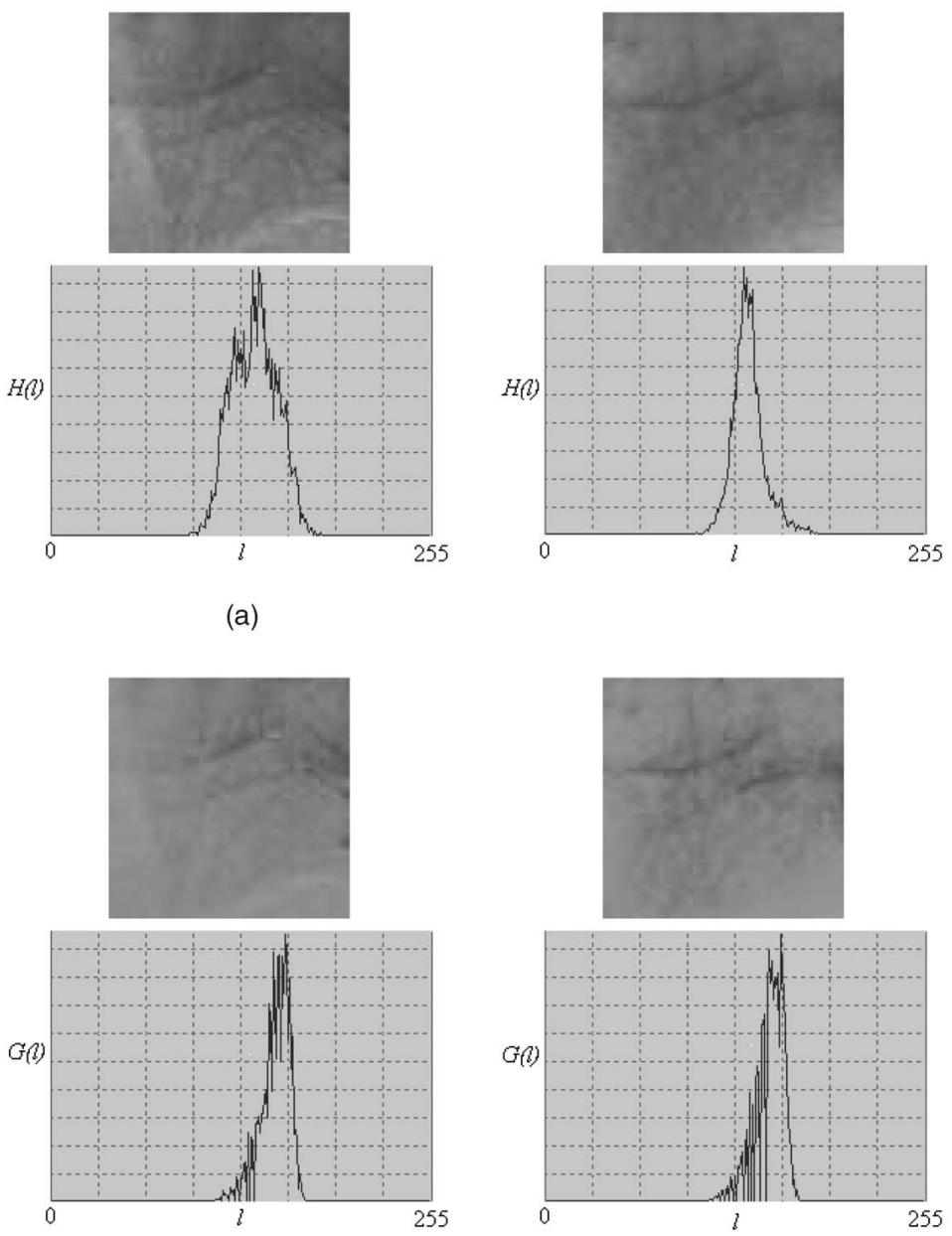

$$
\text { ss }
$$

(a)

(b)

Fig. 6. Palm subimages with the corresponding histograms (a) before and (b) after the histogram fitting.

individual palm and finger subimage can be represented exactly in terms of a linear combination of the eigenvectors. These eigenvectors can be thought of as a set of features that together characterize the variation between the images, without requiring that they correspond to our intuitive notions of palm and finger features.

The dimensionality of each of the six subspaces is selected based on the results of the preliminary recognition experiments on the database described in Section 6.1. The
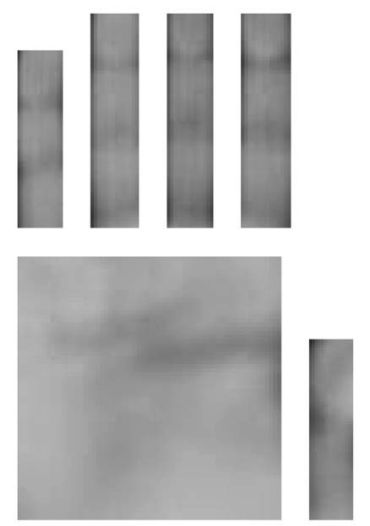

Fig. 7. Average palm and finger images from our database. 


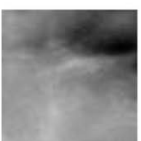

$\mathrm{i}=1$

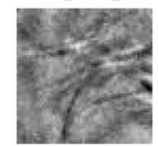

40

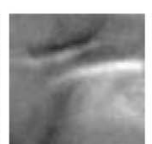

2

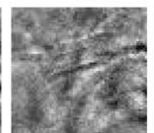

50

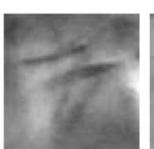

3

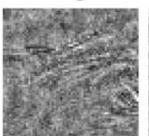

100

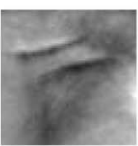

4

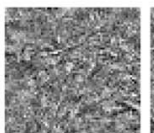

150

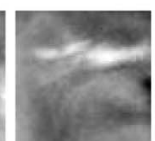

5

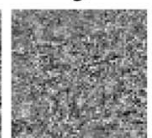

200

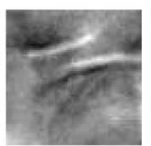

10

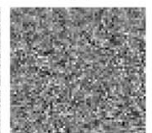

300

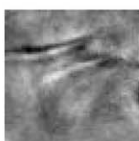

20

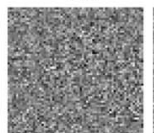

400

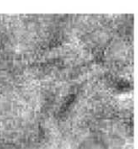

30

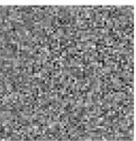

500

(a)
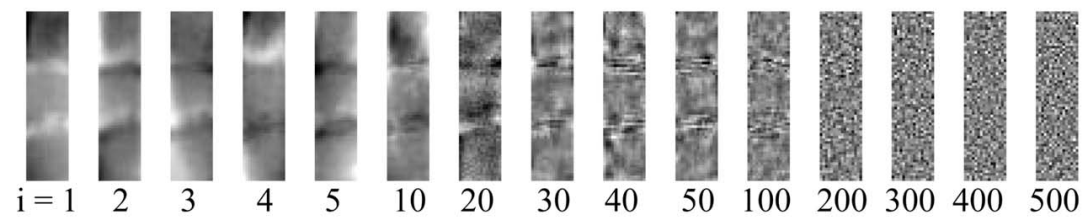

$0 \begin{array}{llll}200 & 300 & 400 & 500\end{array}$

(b)

Fig. 8. Eigenpalms and eigenfingers obtained from the database (indicated by the corresponding ordinal numbers): (a) the eigenpalms and (b) the little-finger eigenfingers.

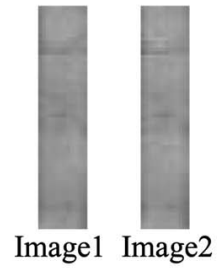

(a)

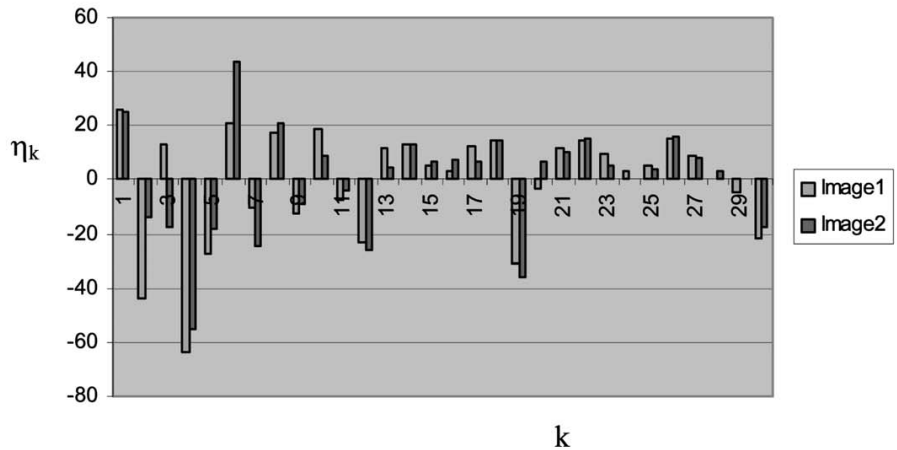

(b)

Fig. 9. (a) Middle-finger subimages of the same person. (b) The first 30 features obtained from the above images.

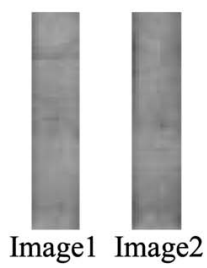

(a)

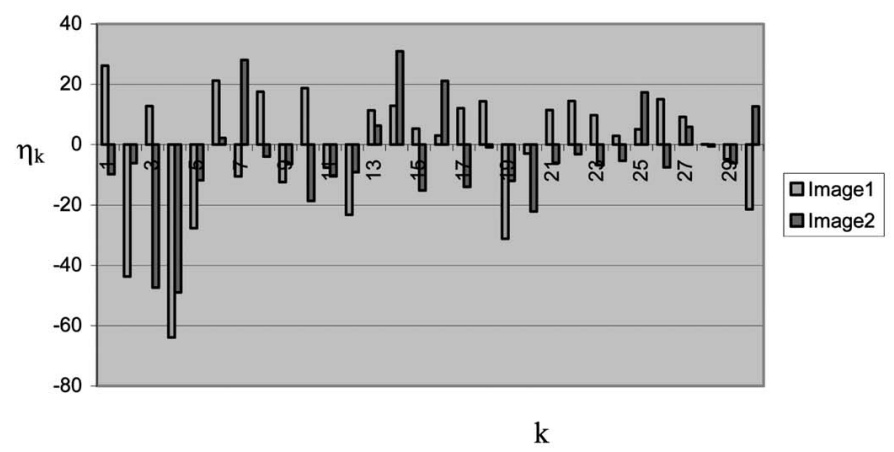

(b)

Fig. 10. (a) Middle-finger subimages of the different people. (b) The first 30 features obtained from the above images.

\section{Matching and Fusion at the MATCHING-SCORE LEVEL}

A template in our system is represented by six feature vectors: palm feature vector $\mathbf{P}_{\mathbf{x}}$ and five finger-feature vectors $\mathbf{F}_{\mathbf{x}}^{\mathbf{i}} ; \mathrm{i}=1,2, \ldots 5$.

Figs. 9 and 10 demonstrate why including finger-strip features as discriminatory features in a matching process is justified. They show the first $k, k<m$ features $\eta_{k}$ obtained from the middle-finger subimages.
In order to recognize, identify, or verify a user, the matching process between the live-template and the templates from the database has to be performed. The matching between corresponding feature vectors is based on the Euclidean distance. In this step, the following six distances are obtained:

- $\mathrm{d}\left(\mathbf{P}_{\mathbf{x}}, \mathbf{P}_{\mathbf{j}}\right)$; where $\mathbf{P}_{\mathbf{x}}$ is a palm live-template and $\mathbf{P}_{\mathbf{j}}, \mathbf{j}=$ $1,2, \ldots u$ are palm templates from the database, where $u$ is the total number of templates in the database. 
TABLE 1

The Values of $\mu$ and $\tau$ for Palm and Fingers

\begin{tabular}{|c|c|c|c|c|c|c|}
\hline & palm & $\begin{array}{c}\text { little } \\
\text { finger }\end{array}$ & $\begin{array}{c}\text { ring } \\
\text { finger }\end{array}$ & $\begin{array}{c}\text { middle } \\
\text { finger }\end{array}$ & $\begin{array}{c}\text { index } \\
\text { finger }\end{array}$ & thumb \\
\hline$\mu$ & 347 & 142 & 90 & 92 & 149 & 193 \\
\hline$\tau$ & 680 & 300 & 240 & 220 & 320 & 330 \\
\hline
\end{tabular}

- $\mathrm{d}\left(\mathbf{F}_{\mathbf{x}}^{\mathbf{i}}, \mathbf{F}_{\mathbf{j}}^{\mathbf{i}}\right) ; \mathrm{i}=1,2, \ldots 5$, where $\mathbf{F}_{\mathbf{x}}^{\mathbf{i}}$ are finger livetemplates and $\mathbf{F}_{\mathrm{j}}^{\mathbf{i}}, \mathrm{j}=1,2, \ldots u$ are finger templates from the database.

The distances are normalized and transformed into similarity measures $\mathrm{S}_{\mathrm{xj}}^{\mathrm{P}}$ and $\mathrm{S}_{\mathrm{xj}}^{\mathrm{Fi}} ; \mathrm{i}=1,2, \ldots 5$, by means of six transition functions $\left(\mathrm{S}^{\mathrm{P}}\right.$ and $\left.\mathrm{S}^{\mathrm{Fi}} ; \mathrm{i}=1,2, \ldots 5\right)$, which were determined experimentally from the training set of the database as follows:

The frequency distribution of the distances $d\left(\mathbf{P}_{\mathbf{p}}, \mathbf{P}_{\mathbf{j}}\right)$, and $\mathrm{d}\left(\mathbf{F}_{\mathbf{p}}^{\mathbf{i}}, \mathbf{F}_{\mathbf{j}}^{\mathbf{i}}\right) ; \mathrm{i}=1,2, \ldots 5$, where $p$ and $j$ denote templates of the same person, is calculated. This process is performed for every set of user templates from the training set of the database. The corresponding frequency distributions are collected for all users, and a histogram $\mathrm{H}(\mathrm{d})$ is obtained.

The transition functions are three-segment functions over the distances interval $[0,+\infty)$. The first segment is a constant function with the value 1 , over the interval $[0, \mu)$, where $\mu$ is the mean value of the distances. The second segment is a linear function that approximates the histogram's right-hand tail over the interval $[\mu, \tau)$, where $\mathrm{H}(\tau)=0$. The third segment is a constant function with the value 0 , over the interval $[\tau,+\infty)$. Table 1 shows the values of $\mu$ and $\tau$ for the palm and fingers obtained automatically during the training phase. Fig. 11 illustrates a transition function $\mathrm{S}^{\mathrm{P}}$, based on the frequency distribution of the palm distances.

The normalized outputs of the six matching modules are combined using fusion at the matching-score level. The fusion is expressed by means of the total similarity measure $\left(\mathrm{TSM}_{\mathrm{xj}}\right)$

$$
\begin{aligned}
T S M_{x j}= & w_{1} \cdot S_{x j}^{P}+w_{2} \cdot S_{x j}^{F 1}+w_{3} \cdot S_{x j}^{F 2} \\
& +w_{4} \cdot S_{x j}^{F 3}+w_{5} \cdot S_{x j}^{F 4}+w_{6} \cdot S_{x j}^{F 5},
\end{aligned}
$$

where $w_{1}, w_{2}, \ldots, w_{6}$ are weight factors associated with each of the hand parts and fulfill the condition $w_{1}+w_{2}+$ $\ldots+w_{6}=1$. In our system, the weights are set proportionally to the preliminary unimodal recognition results (see Section 6.1) So that $w_{1}=0.169, w_{2}=0.168, w_{3}=0.174$, $w_{4}=0.178, w_{5}=0.163$, and $w_{6}=0.146$.

\section{EXPERIMENTAL RESULtS}

\subsection{Recognition}

It is known that the $\mathrm{K}-\mathrm{L}$ transform finds components that are useful for representing data, and there are no well-founded reasons, in general, to assume that these components are useful for discriminating between data in different classes [42], i.e., the K-L transform seeks directions that are efficient for the representation of data in a least-squares' sense.

The preliminary recognition experiments carried out and described in this section have to answer the following questions: 1) Are the K-L components for the six subspaces

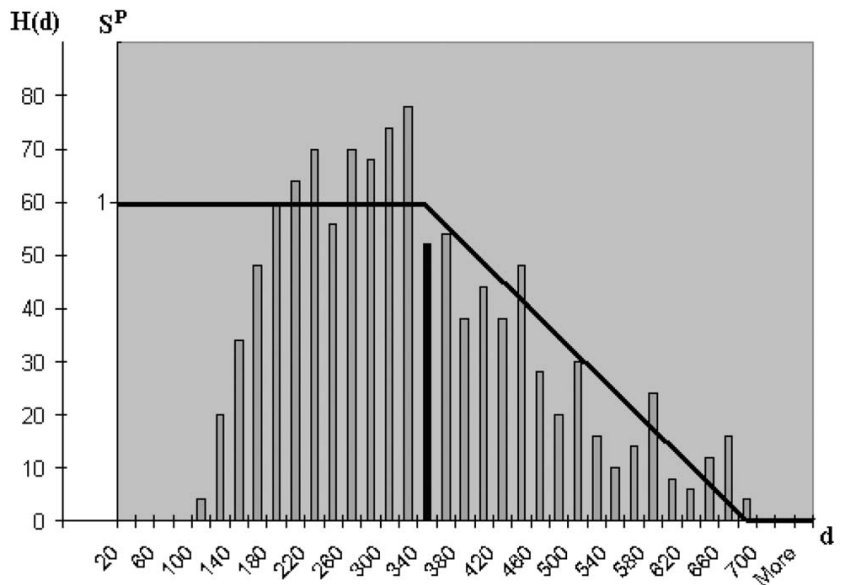

Fig. 11. Distance to the similarity transition function based on the frequency distribution of the palm distances $(\mu=347, \tau=680)$.

(subspaces of the palm and fingers) sufficiently discriminatory for recognition purposes? 2) What is the dimensionality of the subspaces that gives a satisfactory recognition accuracy?

To answer the first question, we made some simple preliminary experiments that showed the recognition results achieved using the features from different hand parts. We also demonstrated how fusion influences the recognition accuracy.

Seven recognition experiments were made. Six of them were made using features from only one hand part (i.e., recognition based only on eigenpalm features, recognitionbased only on little-finger eigenfinger features, etc.). The final experiment was done using all the features and fusion at the matching-score level as described in Section 5 . The recognition experiments based on features from only one feature set (eigenpalm or eigenfinger) were conducted as follows:

To begin with, we have a template database consisting of 550 templates (110 users, 5 templates per user). We take the first template from the database and separate it from the rest. This template is then matched to all the templates remaining in the database. A very simple criterion of minimum Euclidean distance is used for the recognition. After this, the separated template is returned to the database, the next template is separated, and the process described above is repeated until every template in the database is matched with the remainder of the database. The above process is repeated for the templates representing each part of the hand.

The recognition experiment in which all the features were used was conducted in a similar manner, except that fusion at the matching-score level was used. Instead of minimum Euclidean distance, a maximum total similarity measure was used as the recognition criterion. The recognition results for all seven experiments are shown in Fig. 12.

It is clear from the results shown in Fig. 12 that a satisfactory recognition result can be achieved with each of the hand parts used. What is particularly surprising is the very high recognition accuracy for some of the fingers, since strip-like finger images like those used in our system have never been considered in biometric systems before-as far as we know.

It is also clear that fusion at the matching-score level in the recognition process significantly improves its accuracy. 


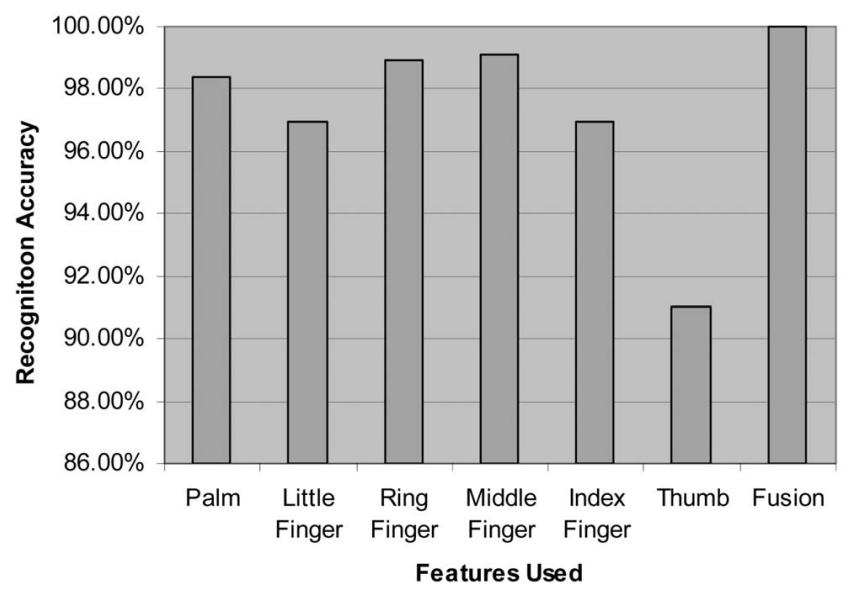

Fig. 12. Recognition results based on different hand parts and their fusion.

With our database, when fusion was used for the recognition, a perfect score (100 percent) was achieved.

The answer to the question about the satisfactory dimensionality of the feature spaces can be obtained by experiments in which the unimodal recognition results are analysed as a function of the subspace dimensionality. Fig. 13 shows the results of the experiments.

Fig. 13 demonstrates that a high recognition accuracy was achieved for the palm subspace dimensionality $\mathrm{m}_{\mathrm{P}}=150$ and the finger subspaces dimensionality $\mathrm{m}_{\mathrm{F}}=100$. Similar results for the palm only were obtained in [30].

\subsection{Identification}

We created a database of 1,820 hand images (samples) of 237 people. The entire database was collected over the period of six months. The interval over which the samples of individual persons were acquired varied, with some persons giving all samples at one time, and the others over the period of up to one month. The early preliminary experiments carried out in order to verify the permanence of the used biometric features showed us that they do not change significantly over such period of time, even for the people involved in physical activities potentially stressful for the regions (i.e., tennis-players). Two identification experiments were done in order to test the performance of the system. Fivehundred fifty samples of 110 people (five samples per person) were used as a training set. The training set was used for generation of finger and palm eigen-basis, determination of transition functions $\mathrm{S}^{\mathrm{Fi}} ; \mathrm{i}=1,2, \ldots, 5$ and $\mathrm{S}^{\mathrm{P}}$, and finally, obtaining the weight factors $\mathrm{w}_{\mathrm{i}} ; \mathrm{i}=1,2, \ldots, 6$. The 110 people whose samples were collected for the training set were not involved, neither as the clients nor as the impostors, in the following two experiments.

Experiment 1. The first identification experiment was carried out on a database consisting of two parts: a client database and an impostor database. In this experiment, 57 people were selected to act as clients. Ten samples were acquired per client. The first seven samples from each client were used in the enrolment to generate a client-template database. Seven templates in the database represent each client. The remaining three samples were used for testing. The remaining 70 people (237 persons total - 110 persons used in the training set - 57 clients), 10 samples per person, had the role of impostors. This set-up makes for $57 \times 3=171$ client experiments and $70 \times 10=700$ impostor experiments. Note that the samples used as impostors and clients in the testing phase were not used in the training phase of the system.

The identification experiments were made as follows: When an unknown sample is presented to the identification system, the system generates a live-template and calculates the total similarity measure (TSM) between the live-template and all the templates stored in the client database (there are $57 \times 7=399$ templates). The system uses the $(\mathrm{k}, 1)-\mathrm{NN}$ classifier, $\mathrm{k}=1=3$, to determine the person to whom the unknown sample belongs. This means that it is necessary for the three closest templates (with the largest TSM) from the client database to belong to the same person, for example, the person with index $p$, otherwise the person represented by the live-template is rejected as an impostor.

In the next step, the final decision (the user is identified or rejected) is based on thresholding: if

$$
\max _{i}\left(T S M_{i}\right) \geq T ; i=1,2,3,
$$

then the person represented by the live-template is successfully identified as a user registered in the database with index $p$. Otherwise, the person represented by the livetemplate is rejected as an impostor. Thresholding is a very important step for the elimination of impostors. Preliminary identification experiments based only on the (k,l)-NN rule, without using a threshold gave a FRR (false rejection rate) = 0 percent, but a FAR (false acceptance rate) $=24.4$ percent .

The results, expressed as a FRR, and a FAR, vary, depending on the threshold T. Fig. 14 presents the identification test results and shows the dependency of the FAR and the FRR on the threshold value.

From Fig. 14, it is clear that our identification system achieves an EER (equal error rate) of $F R R=F A R=0.58$ percent for threshold $\mathrm{T}=0.83$. A minimum TER (Total Error Rate, TER $=$ FAR + FRR) of 0.72 percent is achieved with $\mathrm{T}=0.85$.

Our system can achieve a FAR $=0.0$ percent with a FRR $=$ 1.17 percent with $\mathrm{T}=0.87$. It can also achieve a FRR $=$ 0.0 percent with a $\mathrm{FAR}=3.29$ percent with $\mathrm{T}=0.78$.

We also repeated the experiment using the 1-NN classifier. The preliminary results showed that the FRR didn't change at all, while the FAR became increased.

Experiment 2. In this experiment, exhaustive testing of the system was performed as follows: The system was tested on a database of 127 people. Ten samples of each person's hand were captured, thus a total of 1,270 samples were made available. When a person played a role of a client, seven of his / her samples were used in the enrolment phase to create seven client templates; the remaining three samples were used for testing. If a person acted as an imposter, however, all 10 samples were used for testing.

The experiment proceeded as follows: One person was randomly selected from the set of 127 people to act as a client and was enrolled in the database. The remaining 126 people were considered impostors. The identification was performed and the results were recorded. Next, from the 127 people, two of them were randomly selected and declared as the authorized users. So, at this point, there were two clients and 125 impostors. Again, a process of identification was performed and the results were recorded. The above 


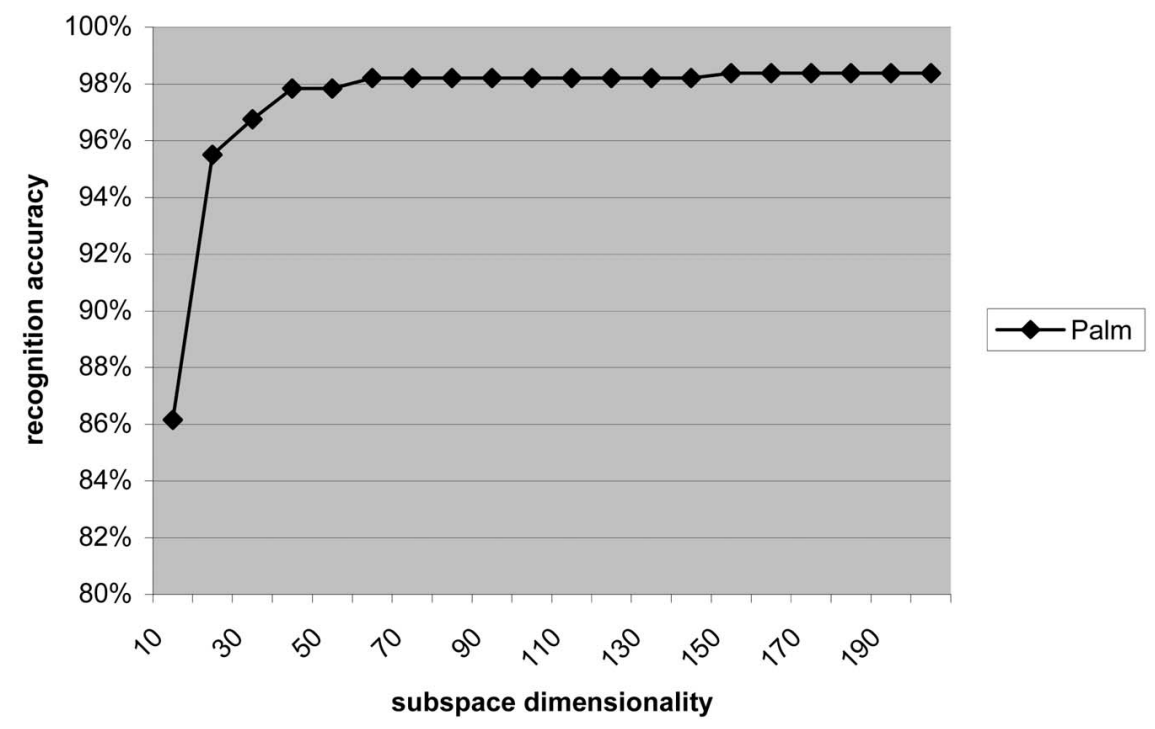

(a)

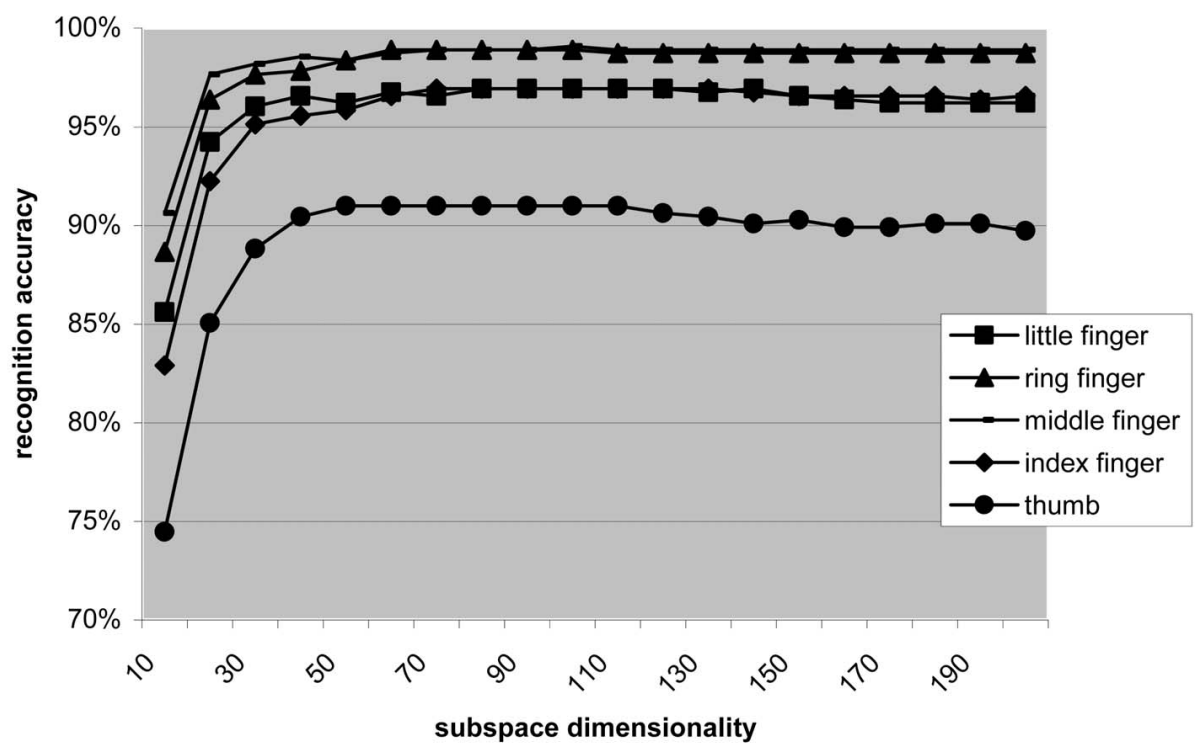

(b)

Fig. 13. Recognition results as a function of the subspace dimensionality for (a) palm subspace and (b) finger subspaces.

procedure was repeated until all 127 people were declared as clients, and no impostor remained. The whole of the abovedescribed process was repeated 20 times. There were a total of more then 1.6 million imposter experiments and more then 480 thousand client experiments.

The experiment showed the EER $=0.75$ percent and minimum TER=1.06 percent. The FAR and FRR, depending on the selected threshold, are shown in Fig. 15.

Identification time. The total identification time per person consists of a fixed time (required for the hand-image preprocessing, subimage extraction, normalization, and projection) and a variable time for the one-to-many matching process:

total_identification_time $=$

fixed_time $+M($ one - to - one_matching_time $)$, where $M$ is the number of all the templates stored during the enrollment process, the fixed_time is 0.544 seconds, and the one-to-one_matching_time is 0.003 milliseconds on a computer with 1177 SPECint_base2000 and 1154 SPECfp_base2000). For example, in Experiment $1(\mathrm{M}=57 \times 7)$ the identification time per person is 0.545 seconds.

\section{Conclusion}

We have developed a prototype of an online biometric identification system based on eigenpalm and eigenfinger features. The experimental results, obtained on a database of 237 people ( 1,820 hand images), show that it achieves a very high recognition rate (100 percent), and an identification (7) accuracy expressed in terms of an equal error rate (EER) of 0.58 percent and a total error rate (TER) of 0.72 percent. 


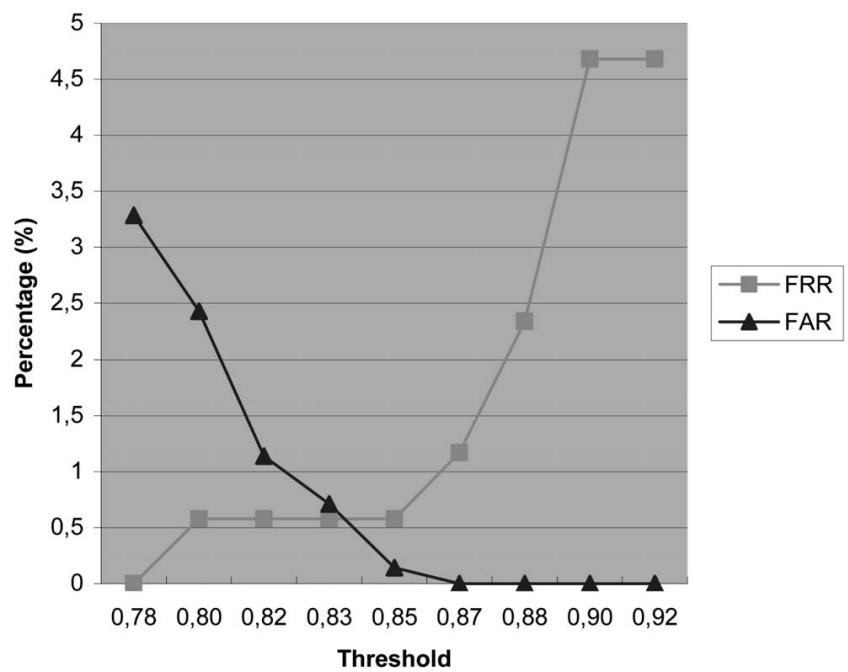

Fig. 14. Identification test results (Experiment 1), the dependence of the FAR and the FRR on the threshold.

Compared with the approaches based on features obtained from the palm described in [9], [20], [21], [22], [30], our system achieves better results expressed in terms of the total error rate or equal error rate.

The total identification time per person (as explained in the Section 6.2) consists of a fixed time (0.544 seconds) and a matching time, which is 0.003 milliseconds per template in the client database (on a computer with 1177 SPECint_base2000 and 1154 SPECfp_base2000). For example, for a database of 1,000 clients ( 7 templates per client), the total identification time would be 0.565 seconds, which is fast enough for realtime identification.

The use of a multimodal approach, rather than concentrating just on the palm area, was shown to be very important for improving the system's accuracy. Strip-like finger images were shown to hold biometric features that can, even by themselves, be used to achieve a good recognition accuracy. It was also shown how fusion at the matching-score level greatly increases the biometric system's accuracy.

In the future, we plan to create a larger hand-images database, with images taken over a longer period of time, and test our system's accuracy on this database. We also believe that the system could be further improved by the fusion of other hand characteristics, such as principal palm lines and hand geometry. We are also going to apply linear and multiple discriminant analysis to find projections in order to compare the obtained results of the identification with the ones obtained by means of the K-L transform.

\section{ACKNOWLEDGMENTS}

This work was supported by the Croatian Ministry of Science, Education, and Sport as a part of the project no. 0036025. The authors would like to thank the anonymous reviewers for their valuable comments and suggestions.

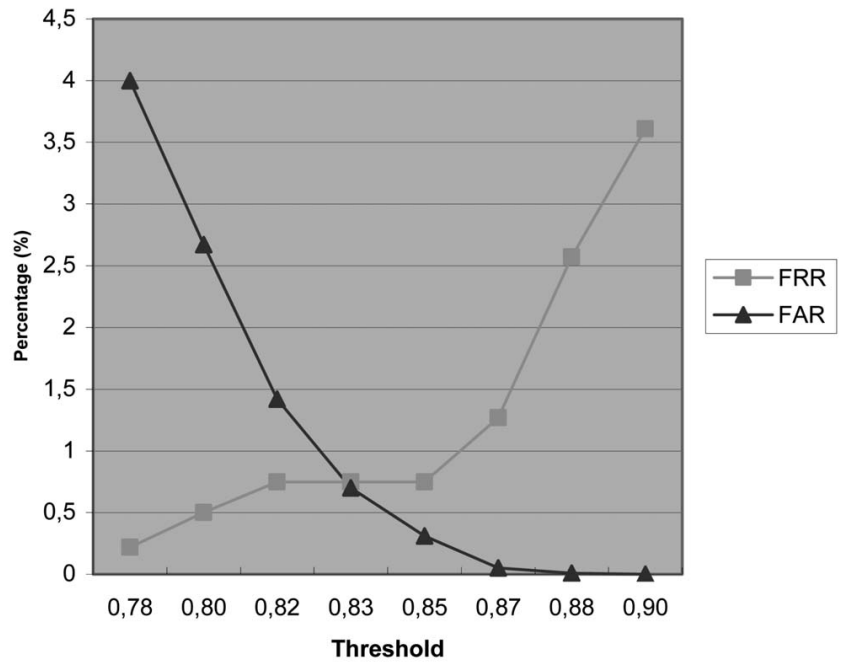

Fig. 15. Identification test results (Experiment 2), the dependence of the FAR and the FRR on the threshold.

\section{RefERENCES}

[1] Biometrics: Personal Identification in Networked Society, A.K. Jain, R. Bolle, and S. Pankanti, eds. Kluwer Academic, 1999.

[2] D. Zhang, Automated Biometrics: Technologies \& Systems. Kluwer Academic, 2000.

[3] A.K. Jain, L. Hong, S. Pankanti, and R. Bolle, "An Identity Authentication System Using Fingerprints," Proc. IEEE, vol. 85, no. 9, pp. 1365-1388, 1997.

[4] Intelligent Biometric Techniques in Fingerprint and Face Recognition, L.C. Jain, U. Halici, I. Hayashi, and S.B. Lee, eds. CRC Press, 1999.

[5] A.K. Jain, A. Ross, and S. Pankanti, "A Prototype Hand GeometryBased Verification System," Proc. Second Int'l Conf. Audio- and Video-Based Biometric Person Authentication, pp. 166-171, Mar. 1999.

[6] R. Sanchez-Reillo, C. Sanchez-Avila, and A. Gonzalez-Marcos, "Biometric Identification through Hand Geometry Measurements," IEEE Trans. Pattern Analysis and Machine Intelligence, vol. 22, no. 10, pp. 1168-1171, Oct. 2000.

[7] W. Shu and D. Zhang, "Automated Personal Identification by Palmprint," Optical Eng., vol. 37, no. 8, pp. 2359-2362, Aug. 1998.

[8] D. Zhang and W. Shu, "Two Novel Characteristics in Palmprint Verification: Datum Point Invariance and Line Feature Matching," Pattern Recognition, vol. 32, no. 4, pp. 691-702, 1999.

[9] D. Zhang, W.K. Kong, J. You, and M. Wong, "Online Palm Print Identification," IEEE Trans. Pattern Analysis and Machine Intelligence, vol. 25, no. 2, pp. 1041-1050, Feb. 2003.

[10] J. Zhang, Y. Yan, and M. Lades, "Face Recognition: Eigenface, Elastic Matching, and Neural Nets," Proc. IEEE, vol. 85, no. 9, pp. 1423-1435, 1997.

[11] R.P. Wildes, "Iris Recognition: An Emerging Biometric Technology," Automated Biometrics: Technologies \& Systems, pp. 1348-1363, 2000.

[12] M. Negin, T.A. Chmielewski Jr., M. Salganicoff, T.A. Camus, U.M. Cahn von Seelen, P.L. Venetianer, and G.G. Zhang, "An Iris Biometric System for Public and Personal Use," Computer, vol. 33, no. 2, pp. 70-75, Feb. 2000.

[13] R. Hill, "Retina Identification," Biometrics: Personal Identification in Networked Society, A.K. Jain, R. Bolle, and S. Pankanti, eds., Kluwer Academic, pp. 123-141, 1999.

[14] M. Burge and W. Burger, "Ear Biometrics," Biometrics: Personal Identification in Networked Society, A.K. Jain, R. Bolle, and S. Pankanti, eds., Kluwer Academic, pp. 273-286, 1999.

[15] C. Bregler and Y. Konig, "Eigenlips for Robust Speech Recognition," Proc. IEEE Int'l Conf. Acoustics, Speech, and Signal Processing, pp. 669-672, 1994.

[16] J.P. Campbell, "Speaker Recognition: A Tutorial," Automated Biometrics: Technologies \& Systems, pp. 1437-1462, Kluwer Academic, 2000.

[17] A.K. Jain and A. Ross, "Multibiometric Systems," Comm. ACM, vol. 47, no. 1, pp. 34-40, Jan. 2004. 
[18] Int'1 Committee for Information Technology Standards, Technical Committee M1, Biometrics, http://www.incits.org/tc_home/ m1.htm, 2005.

[19] "A Performance Evaluation Of Biometric Identification Devices," The 1991 Sandia Report, Report summary prepared by Recognition Systems, Inc., http:/ /www.login.hu/termekek/rsi/whitepapers / sandiareport.htm, 1991.

[20] N. Duta, A.K. Jain, and K.V. Mardia, "Matching of Palmprints," Pattern Recognition Letters, vol. 23, no. 4, pp. 477-485, 2001.

[21] J. You, W. Li, and D. Zhang, "Hierarchical Palmprint Identification via Multiple Feature Extraction," Pattern Recognition, vol. 35, no. 4 , pp. 847-859, 2002.

[22] C.C. Han, H.L. Cheng, K.C. Fan, and C.L. Lin, "Personal Authentication Using Palmprint Features," Pattern Recognition, vol. 36, no. 2, pp. 371-381, 2003.

[23] M. Golfarelli, D. Maio, and D. Maltoni, "On the Error-Reject Trade-Off in Biometric Verification Systems," IEEE Trans. Pattern Analysis and Machine Intelligence, vol. 19, no. 7, pp. 786-796, July 1997.

[24] A.K. Jain and N. Duta, "Deformable Matching of Hand Shapes for User Verification," Proc. Int'l Conf. Image Processing, pp. 857-861, 1999.

[25] Recognition Systems Inc, http://www.recogsys.com, 2005.

[26] NEC Automated PalmPrint Identification System http:// www.necsolutions-am.com/idsolutions/download/automated palmprint_id_system.pdf, 2005.

[27] Printrak Automatic Palmprint Identification System, http:// www.printrakinternational.com, 2001.

[28] M. Turk and A. Pentland, "Eigenfaces for Recognition," J. Cognitive Neuroscience, vol. 3, no. 1, pp. 71-86, Mar. 1991.

[29] Y. Hamada, N. Shimada, and Y. Shirai, "Hand Shape Estimation Using Image Transition Network," Proc. Workshop Human Motion, pp. 161-166, 2000.

[30] G. Lu, D. Zhang, and K. Wang, "Palmprint Recognition Using Eigenpalms Features," Pattern Recognition Letters, vol. 24, nos. 9-10, pp. 1463-1467, 2003.

[31] L. Hong and A.K. Jain, "Multimodal Biometrics," Automated Biometrics: Technologies \& Systems, pp. 326-344, Kluwer Academic, 2000.

[32] A. Ross and A. Jain, "Information Fusion in Biometrics," Pattern Recognition Letters, vol. 24, no. 13, pp. 2115-2125, 2003.

[33] S. Ribaric, D. Ribaric, and N. Pavešić, "Multimodal Biometric User-Identification System for Network-based Applications," IEE Proc. Vision, Image \& Signal Processing, vol. 150, no. 6, pp. 409-416, Dec. 2003.

[34] A. Kumar, D.C.M. Wong, H.C. Shen, and A.K. Jain, "Personal Verification Using Palmprint and Hand Geometry Biometric," Proc. Fourth Int'l Conf. on Audio- and Video-Based Biometric Person Authentication, pp. 668-678, June 2003.

[35] A.K. Jain and A. Ross, "Learning User-Specific Parameters in a Multibiometric System," Proc. of Int'l Conf. Image Processing, pp. 5760, Sept. 2002.

[36] R. Brunelli and D. Falavigna, "Personal Identification Using Multiple Cues," IEEE Trans. Pattern Analysis and Machine Intelligence, vol. 17, no. 10, pp. 995-966, Oct. 1995.

[37] A.K. Jain, S. Prabhakar, and S. Chen, "Combining Multiple Matchers for a High Security Fingerprint Verification System," Pattern Recognition Letters, vol. 20, no. 11-13, pp. 1371-1379, 1999.

[38] J. Kittler, M. Hatef, R.P.W. Duin, and J. Matas, "On Combining Classifiers," IEEE Trans. Pattern Analysis and Machine Intelligence, vol. 20, no. 3, pp. 226-239, Mar. 1998.

[39] J. Kittler and F.M. Alkoot, "Sum versus Vote Fusion in Multiple Classifier Systems," IEEE Trans. Pattern Analysis and Machine Intelligence, vol. 25, no. 1, pp. 110-115, Jan. 2003.

[40] R.C. Gonzales and R.E. Woods, Digital Image Processing. Addison Wesley, 1993.

[41] W.H. Press, S.A. Teukolsky, W.T. Vetterling, and B.P. Flannery, Numerical Recipes in C, the Art of Scientific Computing, second ed. Cambridge Univ. Press, 1992.

[42] R.O. Duda, P.E. Hart, and D.G. Stork, Pattern Classification. New York: J. Wiley, 2001.

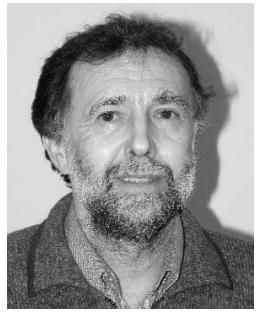

Slobodan Ribaric received the BS degree in electronics, the MS degree in automatics, and the $\mathrm{PhD}$ degree in electrical engineering from the Faculty of Electrical Engineering, Ljubljana, Slovenia, in 1974, 1976, and 1982, respectively. $\mathrm{He}$ is currently a full professor in the Department of Electronics, Microelectronics, Computer, and Intelligent Systems, Faculty of Electrical Engineering and Computing, University of Zagreb, Croatia. His research interests include pattern recognition, artificial intelligence, biometrics, computer architecture, and robot vision. He is author of four books (Microprocessor Architecture, The Fifth Computer Generation Architecture, Advanced Microprocessor Architectures, and CISC and RISC Computer Architecture) and coauthor of one (An Introduction to Pattern Recognition). He is a member of the IEEE, IEEE Computer Society, ISAI, and IAPR.

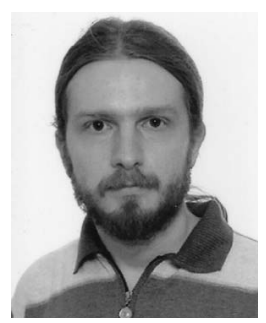

Ivan Fratric received the BS degree in computing from the University of Zagreb in 2003. He is currently a $\mathrm{PhD}$ student in the Department of Electronics, Microelectronics, Computer, and Intelligent Systems at the Faculty of Electrical Engineering and Computing at the same university. His research interests include machine vision, pattern recognition, biometrics, and artificial intelligence.

$\triangleright$ For more information on this or any other computing topic, please visit our Digital Library at www.computer.org/publications/dlib. 